\title{
Labyrinthe
}

$10 \mid 2001$

Numéro 10

\section{Le lien de famille : unicité ou pluralité ?}

\section{Emmanuelle Roman}

\section{(2) OpenEdition}

\section{Journals}

Édition électronique

URL : http://journals.openedition.org/labyrinthe/1197

DOI : $10.4000 /$ labyrinthe. 1197

ISSN : 1950-6031

Éditeur

Hermann

Édition imprimée

Date de publication : 1 octobre 2001

Pagination : 39-52

Référence électronique

Emmanuelle Roman, "Le lien de famille : unicité ou pluralité ? », Labyrinthe [En ligne], 10 | 2001, mis en ligne le 07 avril 2006, consulté le 06 mai 2019. URL : http://journals.openedition.org/labyrinthe/1197 ; DOI : 10.4000/labyrinthe. 1197

Ce document a été généré automatiquement le 6 mai 2019.

Propriété intellectuelle 


\title{
Le lien de famille : unicité ou pluralité?
}

\author{
Emmanuelle Roman
}

1 Le droit détermine la famille à partir du groupe, de l'ensemble des personnes unies par un lien de parenté ${ }^{1}$. Aussi le lien de famille, qui réalise l'attachement d'une personne à son ascendant ou à son descendant, est direct. Le lien de famille indirect désigne la collatéralité ${ }^{2}$. En d'autres termes, la famille est définie soit en un sens large comme l'ensemble des personnes descendant d'un auteur commun unies par un lien de parenté ou de filiation, soit en un sens étroit comme l'ensemble des époux et de leurs enfants mineurs ${ }^{3}$. La famille, appréhendée dans un sens étroit, ou famille nucléaire, retient plus particulièrement notre attention. En effet, le lien de famille, qu'elle consacre, est le plus révélateur des changements socio-juridiques intervenus en son sein.

2 Mais au-delà de la juste théorisation du concept de famille et, a fortiori, du concept de lien de famille, on ne peut aujourd'hui que constater la complexité du phénomène familial.

3 La menée de programmes publics sur le lien de famille témoigne de cette complexité. À titre d'illustration, le 4 avril 2001, le Ministre de la Justice et le Ministre délégué à l'Enfance, à la Famille et aux Personnes handicapées, ont présenté le contenu du document d'orientation sur le droit de la famille. Or, la finalité de ce document, précédant une future révision du droit de la famille, est justement de réformer le sens de l'institution familiale tout en respectant la diversité des familles ${ }^{4}$. De même, Irène Théry, sociologue de la famille et auteur d'un rapport officiel sur le couple, la filiation et la parenté, évoque concrètement l'idée de mutation de la cellule familiale ${ }^{5}$, cette dernière ayant fait preuve d'une perméabilité manifeste face à la multiplication des choix individuels de vie. Enfin, les chiffres récents de 2000, mesurant les transformations du fait familial, confortent ces observations. Selon une source INSEE, et en retenant les chiffres les plus révélateurs de l'évolution du lien de famille, on a recensé $47 \%$ de couples non mariés avec enfants contre $31 \%$ en 1980. Parallèlement, les naissances hors mariage se sont élevées sensiblement au rang de 40,7 \% contre $11,4 \%$ en $1980^{6}$.

Or, quelle est la réponse de l'institution, du droit, face à cet état du fait familial? Comment peut-on définir aujourd'hui le lien de famille? Qu'est ce qui fait le lien de famille au regard du législateur ? N'est-il qu'un ou cède-t-il à la pluralité ? Représente-t-il 
le tout pluralisme familial légal ou un pluralisme familial légal raisonné ? En d'autres termes, est-ce que les normes juridiques du droit de la famille épousent de façon systématique les normes sociales familiales?

5 Face à ce questionnement, le champ d'application de cette étude, tentant de circonscrire le lien de famille, a dû être déterminé à la fois d'une manière générale et détaillée. En effet, l'étude porte sur le droit français de la famille, tel qu'il est issu du Code civil ${ }^{7}$ aux titres V, VI, VII, VIII, et IX traitant respectivement du mariage, du divorce, de la filiation, de la filiation adoptive et de l'autorité parentale. Mais l'approche d'autres dispositions légales, car consacrées par le Code de Santé publique ou par le Code de la Sécurité sociale, s'est imposée en raison de l'intérêt que ces règles de droit portent également au lien de famille. D'autres éléments d'information, comme plus proches de la sociologie juridique, ont aussi été pris en considération. Ils apportent un éclairage des plus riches à l'essai de délimitation du lien de famille.

6 Ainsi, à l'intérêt théorique de cette étude répond spontanément un intérêt pratique d'ordre sociétal. Sous un angle théorique, cette approche du lien de famille permet de ne plus opposer le droit de la famille à la sociologie. On observe bien au contraire une correspondance entre les données juridiques et les données sociologiques. De plus, l'étude met surtout en relief les rapports que la société française contemporaine entretient avec la cellule familiale sous toutes ses formes.

7 Par conséquent, ce mouvement d'alternance entre l'évolution sociologique du lien de famille et les interventions multiples du législateur pour rénover le droit de la famille ne peut qu'attester la difficulté de tracer une frontière entre le lien de famille et le non-lien de famille. Il n'en demeure pas moins que si le pluralisme légal familial s'annonce désordonné lorsqu'il s'agit d'offrir un cadre d'accueil au lien de famille, il va s'affirmant quand il s'agit de déterminer les formes par lesquelles le lien de famille peut être juridiquement représenté.

8 Il est délicat d'affirmer que le pluralisme familial légal existe de façon inconditionnelle. En effet, le législateur a opté pour une politique familiale distributive lorsqu'il s'agit de déterminer, pour le lien de famille, une structure institutionnelle d'accueil. Aussi il fait tour à tour preuve de souplesse, en créant le concept juridique de couple hétérosexuel, et de rigidité lorsqu'il ne fait produire aucun effet de droits familiaux à la reconnaissance légale du concubinage.

9 L'émergence, dans le cadre du paysage juridique familial, du concept de couple hétérosexuel célèbre la formation de nouveaux liens de famille. Ils sont nouveaux en ce sens qu'ils ne se définissent plus à partir d'un statut juridique, et, notamment, d'un statut conjugal, mais à partir d'un principe général d'hétérosexualité. La différence de sexes respectée, le législateur avalise en conséquence les familles concubines et les familles recomposées après un divorce ou après la rupture d'une union libre.

10 Ce sont les lois bioéthiques du 29 juillet 1994 qui célèbrent pour la première fois la notion de couple hétérosexuel ${ }^{8}$. On intègre ainsi un cadre familial bien particulier puisqu'il s'agit de permettre à des couples stériles d'accéder au rang de la parenté par l'accès à des techniques de procréation artificielle faisant recours, pour la plupart d'entre elles, à un tiers donneur. Ainsi, l'article L. 152-2 du Code de la Santé publique, issu de cette réforme, dispose en son alinéa 1 que « l'assistance médicale est destinée à répondre à la demande parentale d'un couple » qui, selon l'alinéa 3 suivant, doit être formé d'un homme et d'une femme. On remarque toute la souplesse de cette formule légale. Elle permet effectivement 
de ne pas s'attarder sur le statut juridique du couple intéressé, en d'autres termes, sur le fait de savoir si les deux membres de ce couple sont unis ou non par le lien du mariage.

Dès 1994, le législateur reconnaît donc implicitement le lien de famille naturelle. Plus précisément, il reconnaît un lien de famille volontaire.

En effet, en exigeant uniquement la différence des sexes, le droit se montre ouvert à d'autres choix de vie familiale où le lien de famille à créer ne se caractérise plus systématiquement par sa légitimité. Bien au contraire, il se définit à partir d'une communauté de vie préexistante, propre au couple demandeur', et d'une communauté d'affection annoncée parce qu'elle s'établira entre le couple et l'enfant, né à l'aide de la technique de procréation médicalement assistée. Par conséquent, le législateur reconnaît ouvertement la formation et l'existence d'un lien de famille où la volonté émise par un couple de former une cellule parentale est prépondérante. La norme familiale juridique de la différence des sexes se situe aux antipodes des normes familiales traditionnelles structurelles. En effet, elle cautionne des liens de famille où l'affection et le désir parental ont occupé une place prépondérante dans leur constitution.

Être un parent aujourd'hui implique de garantir à l'enfant un lien qui durera quoi qu'il arrive. Aimer, protéger, soutenir l'enfant, dans toutes les circonstances, qu'il soit beau ou non, intelligent ou non, délinquant ou non, c'est finalement ce qui est attendu aujourd'hui d'un bon parent ${ }^{10}$.

13 Le législateur de 1994 a donc souhaité que le rôle de parent ne soit plus seulement représenté sous l'angle juridique du couple marié. C'est pourquoi seul le concept de couple hétérosexuel a permis de faire abstraction de la différence établie entre ce qui est légitime et ce qui ne l'est pas, en somme, entre ce qui pouvait aspirer à une famille et ce qui ne restait que hors-famille. À la distinction couple marié/couple non marié s'est alors substituée la règle de l'acceptation de la différence familiale. Par voie de conséquence, le droit est aujourd'hui attaché au rôle sexué de père et de mère. Or, cet attachement, selon Éric Fassin, sociologue, présente également l'avantage d'une ouverture vers la modernité, non seulement vers les familles issues du concubinage, mais aussi vers les familles recomposées ${ }^{11}$. En effet, dans cette dernière situation familiale, la différence des sexes est respectée à deux niveaux, à un niveau parental puisque même après un divorce l'enfant est toujours lié à ses père et mère d'origine, et à un niveau beau-parental. La règle de la différence des sexes fédère en quelque sorte les premiers et les seconds liens de famille. La crise vécue par le modèle du couple légitime a sans aucun doute amené le législateur à faire preuve de souplesse y compris dans cette partie technique du droit de la famille où le lien de filiation est établi entre un couple et un enfant né grâce à une assistance médicale à la procréation. Il faut effectivement rappeler qu'un couple marié sur trois divorce et que le lien de famille conjugale ne pouvait continuer à représenter un diktat social. De plus, on perçoit une volonté d'accentuer le pluralisme familial légal au regard du dépôt des nouvelles propositions de réforme du droit de la famille. En effet, il s'agit à présent de faire disparaitre très symboliquement les notions de filiation légitime et de filiation naturelle, et, en outre, d'abroger les discriminations dont sont victimes les enfants adultérins ${ }^{12}$.

14 Néanmoins, ce qui constitue une avancée vers le pluralisme familial légal a été contrebalancé par la réforme du 15 novembre 1999 instituant le pacte civil de solidarité et le concubinage. En effet, ce même concept juridique de couple hétérosexuel a permis de légitimer l'éviction des couples d'homosexuel(le)s du droit de la famille. 

concubinage en tant qu'institution, ceci en consacrant son insertion au sein du Code civil dans un article 515-8. Aussi l'union libre, jusqu'alors simple réalité sociale, ayant progressé depuis les années soixante-dix ${ }^{13}$, le concubinage devient en 1999 une réalité à dimension légale. De plus, les couples de concubins peuvent adhérer à un statut juridique par la contraction d'un pacte civil de solidarité destiné à organiser leur vie commune ${ }^{14}$, notamment, dans le domaine patrimonial. traditionnelle, coexiste de façon égale avec le concubinage. Mais est-ce à dire que le législateur de 1999 défende un pluralisme familial sans limite? Une seconde lecture du texte de la réforme amène une réponse négative à cette interrogation. Le mariage continue à conserver une suprématie certaine dans le cadre du droit de la famille. En effet, la consécration légale du concubinage n'a pas entraîné parallèlement la reconnaissance de la possibilité, qui lui serait inhérente, de constituer devant la loi un lien de famille. En d'autres termes, le concubinage est devenu une institution civile à défaut de représenter une institution familiale.

Pour conforter cette observation, et avant d'en déterminer la cause, on peut se référer à la décision du Conseil constitutionnel, lequel a été saisi par l'opposition de droite d'un recours pour se prononcer sur la conformité de la réforme à la Constitution. Le 9 novembre 1999, le Conseil constitutionnel déclare que la loi relative au pacte civil de solidarité est conforme à la Constitution. Mais il précise surtout qu'elle «est sans incidence sur les autres titres du livre premier du Code civil, notamment ceux relatifs aux actes d'état civil, à la filiation, à la filiation adoptive et à l'autorité parentale, ensemble de dispositions dont les conditions d'application ne sont pas modifiées par la loi fédérée $»^{15}$. La ligne directrice du Conseil constitutionnel, suivant celle du législateur, est donc de distinguer le lien de couple, que le concubinage représente, du lien de famille, lequel est exclu des dispositions du 15 novembre 1999.

Le législateur a finalement tracé « une démarcation entre le domaine des liens familiaux et celui des affinités choisies " ${ }^{16}$ dans le but de laisser les couples de concubins homosexuels hors du droit de la famille. L'article 515-8 nouveau du Code civil définit effectivement le concubinage comme "une union de fait, caractérisée par une vie commune présentant un caractère de stabilité et de continuité entre deux personnes, de sexe différent ou de même sexe, qui vivent en couple ».

En somme, au regard du contenu de ce texte et de la décision du Conseil constitutionnel, la reconnaissance légale du concubinage n'implique pas que tout couple de concubins, et, notamment, le couple homosexuel, puisse constituer légalement une cellule parentale et mener la vie familiale correspondante. Les couples d'homosexuel(le)s, ayant conclu ou non un pacte civil de solidarité, sont alors dans l'impossibilité juridique de pouvoir adopter un enfant ou d'avoir accès à une technique de procréation artificielle avec tiers donneur. De plus, la contraction d'un pacte civil de solidarité nécessite une déclaration auprès du greffe du tribunal d'instance. Aussi le contrôle, lors d'une requête en adoption, sera des plus renforcés s'il s'agit d'un couple d'homosexuels « pacsés » qui la dépose. À titre de comparaison, le droit français est très éloigné de la nouvelle législation des PaysBas. Par deux lois du 21 décembre 2000, cet État a effectivement ouvert le mariage et la parenté aux couples de personnes de même sexe ${ }^{17}$.

Labyrinthe, 10 | 2006 
20 Pour le législateur il ne peut donc exister un lien de famille homoparentale au sein du droit, lien qui s'établirait légalement à la suite d'une requête en adoption ou d'une demande d'accès à une technique d'assistance médicale à la procréation. C'est pourquoi le modèle du couple hétérosexuel paraît tout à la fois réducteur et libéral : réducteur, parce qu'il ferme la porte du droit de la famille aux personnes de même sexe, libéral, parce qu'il autorise les personnes non mariées à en franchir le seuil. Par le respect du principe de l'hétérosexualité, le législateur accepte, de façon sporadique, la pluralité dans les différentes formes du lien de famille.

21 En optant pour une politique de "saupoudrage ", le législateur défend un pluralisme familial d'essence tacite. Ce sont effectivement des dispositions éparpillées, civiles ou sociales, qui établissent le soutien du droit au lien de famille monoparentale ou au lien de famille pluriparentale.

Le lien de famille monoparentale est « entré dans les mœurs ». En 2000, on dénombre 7,2 $\%$ de familles monoparentales contre 5,3 \% en $1986^{18}$. $13 \%$ des enfants de moins de quinze ans sont élevés en leur sein, dont $88 \%$ avec leurs mères qui exercent le droit de garde ${ }^{19}$. Force est de constater que la monoparentalité ne constitue plus uniquement un phénomène sociétal. Elle se positionne juridiquement aux côtés de la famille traditionnelle, de la famille nucléaire, ceci par l'intermédiaire de deux mesures ponctuelles, l'une appartenant au droit de l'adoption, et l'autre au droit social.

Lié au départ au mouvement féministe des années soixante-dix ${ }^{20}$, le lien de famille monoparentale acquiert dès 1966 une reconnaissance légale. En effet, la réforme majeure du droit de l'adoption du 11 juillet 1966 introduit à l'article 343-1 du Code civil la possibilité pour une personne seule d'adopter en la forme plénière ${ }^{21}$ un enfant. Or, l'adopté se voit attribuer le statut d'enfant légitime. En conséquence, la légitimité n'est plus liée au mariage; elle s'acquiert indépendamment de ce fait institutionnel. Aussi l'explication de cette réforme par le seul motif de la satisfaction du désir d'enfant apparaît-elle trop simpliste. L'article 343-1 du Code civil introduit essentiellement une nouvelle façon de penser le lien de famille. En d'autres termes, le législateur se montre ouvert à une politique du libre choix de la vie familiale.

Ce libéralisme législatif a été accentué par une nouvelle réforme en 1976. Celle-ci crée à l'article 524-1 du Code de la sécurité sociale une allocation spécifique, l'allocation de parent isolé. On a observé que la formation du lien de famille monoparentale correspond moins à un libre choix de vie familiale, mais qu'il s'associe davantage à une situation familiale précaire issue de la dissociation d'une famille conjugale préexistante ${ }^{22}$. Au lieu de rester aveugle à cette détresse familiale, le législateur a donc conforté le lien de famille monoparentale en lui conférant une protection sociale. L'allocation de parent isolé, laquelle a été versée en 2000 à 170000 foyers monoparentaux ${ }^{23}$, s'associe alors, selon les termes du sociologue Jacques Commaille, à « une forme nécessaire et nouvelle de mobilisation du droit ${ }^{24}$.

25 Le droit de la famille ne se contente plus de fédérer, de diriger, des choix familiaux, volontaires ou involontaires. Désormais, il protège ces choix même s'ils s'éloignent de la ligne directrice de la cellule familiale biparentale.

26 En définitive, à deux reprises, en 1966 et en 1976, le législateur a fait figure de concepteur en promouvant une approche nouvelle, car unilinéaire, du lien de famille. Mais si, devant la loi, on peut être un parent " en solitaire $»^{25}$, il apparaît également de moins en moins incongru d'évoquer la notion de pluriparenté en terme juridique. 

d'éducation et de surveillance propres à l'autorité parentale par rapport à la personne de l'enfant. De même, le beau-parent peut garder l'enfant près de lui dans le cas du décès du père ou de la mère biologique, ou conserver, après une séparation, des relations personnelles avec cet enfant. Ces droits impliquent des devoirs, comme celui de participer à l'entretien de l'enfant pendant et après la vie commune, sauf circonstances particulières relevées par le juge. Il va sans dire qu'une telle proposition ne doit devenir effective que lorsque la recomposition est fondée sur des relations de parenté, et non uniquement sur des liens de couple au sein desquels les beaux-pères tiennent seulement " un rôle de compagnonnage conjugal et de simple sociabilité amicale à l'égard des enfants se tenant à l'écart des responsabilités éducatives négociées entre la mère et l'autre parent ${ }^{33}$. 
32 En définitive, deux types de familles recomposées coexistent parallèlement, celles qui sont reconnues par la voie de l'institution de l'enfant du conjoint, et celles qui ne représentent pour le moment qu'une réalité sociétale. Une totale reconnaissance de ce lien de famille implique alors sa prise en considération indépendamment de l'établissement d'un lien d'adoption.

33 Cette observation doit être réitérée lorsque que l'on raisonne en terme de monoparentalité. En conséquence, la pluralité familiale légale implique que le législateur fasse totalement sien le postulat selon lequel vivre en famille revient avant toute autre chose à vivre sous le même toit.

34 En guise de conclusion, on peut émettre la remarque selon laquelle la complexité factuelle du paysage familial actuel se répercute au sein même des normes du droit de la famille, qu'elles soient à dominante civile ou à dominante sociale.

Néanmoins, dans un objectif de simplification théorique, il est intéressant de dresser une typologie des liens de famille à partir du critère de l'opposition juridique lien de famille/ non-lien de famille.

36 Cette typologie regroupe trois catégories de liens de famille. La première catégorie rassemble les liens de famille qui renvoient à une réalité sociale et juridique. Cette juxtaposition des deux réalités s'illustre, à titre d'exemple, par le modèle de la famille nucléaire composée d'une structure biparentale et d'une descendance biologique. La seconde, intermédiaire, regroupe les liens de famille qui correspondent à une réalité sociale et à une réalité juridique de type ponctuel. Il s'agit dans ce cadre de désigner les familles monoparentales et les familles recomposées ou pluriparentales. Enfin, la troisième catégorie rassemble les non-liens de famille. Certes, ils correspondent à une réalité sociale mais celle-ci est dépourvue de toute dimension juridique. Ce sont les familles homoparentales au sein desquelles l'un des membres du couple homosexuel peut élever l'enfant de son compagnon, enfant issu d'une précédente union. De même, ce sont à nouveau les familles recomposées où le beau-parent, n'ayant pas recouru à l'adoption, n'est pas reconnu comme parent par la loi.

Le législateur doit apporter des réponses aux zones de non-droit de l'ordre public familial. Il ne s'agit pas de promouvoir une adaptation illimitée du droit aux mœurs, mais de déterminer des solutions juridiques à la fois protectrices de la cellule familiale et de l'intérêt des personnes les plus vulnérables, ainsi celui de l'enfant. Parmi ces solutions, on privilégie une approche nourricière des relations pouvant se tisser entre un enfant et une personne qui ne peut pas endosser devant le droit positif le rôle de père ou de mère. Le législateur français peut s'inspirer des expériences législatives européennes du lien nourricier ${ }^{34}$. Ainsi, en se préoccupant essentiellement de la vie quotidienne et de l'éducation de l'enfant, le législateur français investirait le tiers nourricier d'une fonction parentale jusqu'à ce que l'enfant ait atteint l'âge de la majorité. L'enfant continuerait à être en contact avec le parent d'origine investi de droits, droit de visite, droit à être informé sur la personne de l'enfant, et de devoirs, comme celui de participer aux frais liés à l'entretien et à l'éducation de l'enfant.

38 Le lien nourricier correspond donc à une approche particulièrement élargie du lien de famille. À notre sens, cette approche confère un nouveau souffle au droit de la famille. En effet, ni lien de filiation, ni lien de parenté mais lien de communauté de vie, le lien nourricier a vocation à s'adapter à de nombreuses situations familiales laissées en suspens par la norme juridique. 


\section{BIBLIOGRAPHIE}

OUVRAGES :

CADOLLE Sylvie, Être parent, être beau-parent. La recomposition de la famille, Paris, Odile Jacob, 2000.

CHAUVIÈRE M., SASSIER M., BOUQUET B. et alii (sous la dir. de), Les Implicites de la politique familiale. Approches historiques, juridiques et politiques, Paris, Dunod, 2000.

DE SINGLY François, Sociologie de la famille contemporaine, Paris, Nathan, coll. « 128 », 1999.

GROsS Martine (sous la dir. de), Homoparentalités, état des lieux. Parentés et différence des sexes, Paris, ESF, coll. « La vie de l'enfant », 2000.

LEROY-Forgeot Flora, MÉCARY Caroline, Le Couple homosexuel et le droit, Paris, Odile Jacob, 2001.

POUSSON-PETIT Jacqueline (sous la dir. de), L'Enfant et les Familles nourricières en droit comparé, Toulouse, Presses de l'université des Sciences sociales de Toulouse, 1997.

SULLEROT Évelyne, Le Grand remue-ménage. La crise de la famille, Paris, Fayard, 1997.

THÉRY Irène, Couple, Filiation et Parenté aujourd'hui. Le droit face aux mutations de la famille et de la vie privée, Rapport à la Ministre de l'Emploi et de la Solidarité et au Garde des Sceaux, Ministre de la Justice, Paris, Odile Jacob/La Documentation française, 1998.

ARTICLES :

SEGALEN Martine, « Grandes tendances », dans L'État de la France 2001-2002, Paris, La Découverte, p. 68-75.

COMMAILlE Jacques, « Famille : entre émancipation et protection sociale », Sciences humaines, avril $2001, n^{\circ} 115$, p. 28-31.

\section{NOTES}

*. Cette étude fait suite à la soutenance d'une thèse de droit privé relative au lien de filiation non biologique, université de Toulon et du Var, 15 décembre 2000, $546 \mathrm{p}$. 1. Gérard Cornu, Vocabulaire juridique, Paris, PUF, 1996, p. 349 ; Catherine Puigelier, Dictionnaire de droit privé, Paris, Centre de publications universitaires, 1999, p. 241.

2. Fille, mère, petite-fille...

3. Frère et sœur qui ont un auteur commun.

4. «Une mise en perspective de la réforme du droit de la famille », Actualités sociales hebdomadaires, 6 avril 2001, n 2209, p. 5.

5. Irène Théry, Couple, Filiation et Parenté aujourd'hui, Le droit face aux mutations de la famille et de la vie privée, Rapport à la Ministre de l'Emploi et de la Solidarité et au Garde des Sceaux, Ministre de la Justice, Odile Jacob/La Documentation française, 1998.

6. Martine Segalen, « Grandes tendances » dans L'État de la France, 2001-2002, Paris, La Découverte, p. 68-69.

7. Code civil, Paris, Dalloz, 2001. 
8. Loi $n^{\circ} 94-653$ du 29 juillet 1994 relative au respect du corps humain, JO, 30 juillet 1994, $\mathrm{n}$ • 175, p. 11056 ; Loi n 94-654 du 29 juillet 1994 relative au don et à l'utilisation des éléments et produits du corps humain, à l'assistance médicale à la procréation et au diagnostic prénatal, JO, ibid., p. 11059.

9. Voir article L. 152-2 du Code de la Santé publique : « L'homme et la femme formant le couple... », et non mariés, « doivent être... en mesure d'apporter la preuve d'une vie commune d'au moins deux ans ».

10. Irène Théry, «La transformation des années soixante/soixante-dix », dans Les Implicites de la politique familiale, Paris, Dunod, 2000, p. 200.

11. Éric Fassin, « La famille "naturalisée” ", dans Les Implicites de la politique familiale, op. cit., p. 226.

12. «Une mise en perspective de la réforme du droit de la famille », op. cit.

13. Évelyne Sullerot, Le Grand remue-ménage, Paris, Fayard, 1997, p. 61-62.

14. Article 515-1 du Code civil.

15. Décision n 99-419 du 9 novembre 1999, JO, 16/11/99, p. 16962-16968.

16. Gérard Bach-Ignasse, Yves Roussel, Le PACS juridique et pratique, Paris, Denoël, 2000, p.

87.

17. Flora Leroy-Forgeot, Caroline Mécary, Le Couple homosexuel et le droit, Paris, Odile Jacob, p. 92 et p. 170.

18. Martine Segalen, op. cit., p. 69-70.

19. Le Monde, 6/07/2001, p. 7.

20. Denis Lensel, Jacques Lafond, La Famille à venir, Une réalité menacée mais nécessaire, Paris, Economica, 2000, p. 64.

21. L'adoption plénière prononce la rupture juridique de l'enfant avec sa famille d'origine, art. 356 du Code civil.

22. Denis Lensel, Jacques Lafond, op. cit., p. 65.

23. Philippe Steck, "L'horlogerie des prestations familiales ", dans Les Implicites de la politique familiale, op. cit., p. 145.

24. Jacques Commaille, «Famille : entre émancipation et protection sociale », Sciences Humaines, avril 2001, n 115, p. 30.

25. Anne Meunier, Le Choix d'adopter, Paris, Flammarion, 1997, p. 132.

26. Irène Théry, « Différence des sexes, Différences des générations », Esprit, décembre 1996, n²27, p. 78.

27. Agnès Martial, « Partages et fraternité dans les familles recomposées », dans Adoptions, Ethnologie des parentés choisies, Paris, La Maison des sciences de l'homme, 1998, p. 207.

28. Irène Théry, « Les constellations familiales recomposées et le rapport au temps : une question de culture et de société ", dans Quels repères pour les familles recomposées ? Paris, LGDJ, 1995, p. 18.

29. Martine Segalen, « Les nouvelles familles ", Sciences humaines, hors-série, $n^{\circ} 26$, septembre-octobre 1999, p. 25.

30. Article 363, alinéa 1 , du Code civil : «L'adoption simple confère le nom de l'adoptant à l'adopté en l'ajoutant au nom de ce dernier ».

31. Sylvie Cadolle, Etre parent, être beau-parent. La recomposition de la famille, Paris, Odile Jacob, 2000, p. 109.

32. Hugues Fulchiron, « Le droit français face au phénomène des recompositions familiales ", dans Quels repères pour les familles recomposées ?, op. cit., p. 135.

33. Sylvie Cadolle, op. cit., p. 80. 
34. Jacqueline Pousson-Petit, L'Enfant et les Familles nourricières en droit comparé, Toulouse, Presses de l'université des Sciences sociales de Toulouse, 1997.

\section{RÉSUMÉS}

Le pluralisme familial légal est présent mais il est caractérisé par le paradoxe. En effet, le mariage ne représente plus l'unique institution à l'origine de la constitution d'un lien de famille. En outre, le lien de famille se décline sous toutes ses formes, de la monoparentalité à la pluriparentalité. À l'opposé, la reconnaissance juridique du concubinage n'a pas impliqué celle des familles homoparentales*.

Family bonds

Kinship relations : unicity or plurality

Legal family plurality exists but in a paradoxical way. Marriage is no longer the unique institution that constitutes kinship. Moreover, with the appearance of single and multiple parenthood, kinship relations have very varied forms. As opposed, the legal recognition of cohabitation without marriage has not implied the same for homosexual parenthood.

\section{AUTEUR}

\section{EMMANUELLE ROMAN}

Emmanuelle Roman est âgée de vingt-huit ans. Elle a poursuivi ses quatre années d'études doctorales auprès de la Faculté de droit de Toulon pour soutenir le 15 décembre 2000 une thèse sur le lien de filiation non biologique. Aujourd'hui, elle poursuit ses recherches, notamment en intervenant sur le secret des origines dans un colloque interdisciplinaire sur le lien, lien social, lien familial. 